Article

\title{
Correlation-Based Robust Authentication (Cobra) Using Helper Data Only
}

\author{
Jim Plusquellic 1,2,* (D) and Matt Areno ${ }^{3}$ \\ 1 Department of Electrical and Computer Engineering, University of New Mexico, Albuquerque, \\ NM 87131, USA \\ 2 IC-Safety, LLC, Albuquerque, NM 87131, USA \\ 3 Trusted and Secure Systems, LLC, Round Rock, TX 78665, USA; matt@trusecsys.com \\ * Correspondence: jimp@ece.unm.edu; Tel.: +1-240-475-1882
}

Received: 3 July 2018; Accepted: 28 August 2018; Published: 31 August 2018

\begin{abstract}
Physical unclonable function (PUF)-based authentication protocols have been proposed as a strong challenge-response form of authentication for internet of things (IoT) and embedded applications. A special class of so called strong PUFs are best suited for authentication because they are able to generate an exponential number of challenge-response-pairs (CRPs). However, strong PUFs must also be resilient to model-building attacks. Model-building utilizes machine learning algorithms and a small set of CRPs to build a model that is able to predict the responses of a fielded chip, thereby compromising the security of chip-server interactions. In this paper, response bitstrings are eliminated in the message exchanges between chips and the server during authentication, and therefore, it is no longer possible to carry out model-building attacks in the traditional manner. Instead, the chip transmits a Helper Data bitstring to the server and this information is used for authentication instead. The server constructs Helper Data bitstrings using enrollment data that it stores for all valid chips in a secure database and computes correlation coefficients (CCs) between the chip's Helper Data bitstring and each of the server-generated Helper Data bitstrings. The server authenticates (and identifies) the chip if a CC is found that exceeds a threshold, which is determined during characterization. The technique is demonstrated using data from a set of 500 Xilinx Zynq 7020 FPGAs, subjected to industrial-level temperature and voltage variations.
\end{abstract}

Keywords: PUF-based authentication; Helper Data correlation; hardware security

\section{Introduction}

Robust authentication and key generation are critically important to defining a root of trust and to providing data integrity and confidentiality in communications between internet-of-things (IoT) devices. Physical unclonable functions (PUFs) are proposed as replacements for traditional non-volatile memory (NVM) for storing keys and for using cryptographic primitives in authentication protocols [1-7]. PUFs are able to reproduce keys and bitstrings on-the-fly by measuring small changes in the signal behavior of an integrated circuit that occur because of the finite, non-zero tolerances that exist in manufacturing processes. A special class of so-called strong PUFs are able to generate uncountable numbers of reproducible bits, making it possible to construct unique response bitstrings for authentication protocols without the need to employ entropy-enhancing cryptographic primitives, such as secure hash, thereby reducing energy and area overheads in IoT devices.

PUF-based authentication protocols that allow direct access to the embedded PUF through an unprotected interface [7], where challenges and responses are not obfuscated using cryptographic primitives, represent the most attractive usage scenario because such protocols are typically very simple and compact. The drawback of protocols with unprotected interfaces is that the embedded 
PUF is susceptible to model-building attacks. Model-building is typically carried out using machine learning (ML) algorithms where the goal of the adversary is build a model of the challenge-response behavior of the PUF and then later use the model to predict the PUF's response to any arbitrary challenge. If this can be accomplished, then the model can be used to impersonate the actual device. Although ML attacks require a large amount of computing effort, they represent a serious threat to PUF-based authentication protocols with unprotected interfaces.

In this paper, we propose a PUF-based, privacy-preserving, mutual authentication protocol with unprotected interfaces that is resilient to model-building attacks. The technique is demonstrated using the hardware-embedded delay PUF (HELP) [7,8] but is applicable to any PUF architecture that is able to produce soft data. Soft data refers to digital values that represent the magnitude of the signal being measured, for example, path delays and frequencies. Soft data captures the inherent, random variations that occur in these signals from one chip to another and represents the source of entropy for the PUF. The proposed methodology can therefore be applied to an enhanced version of the arbiter PUF [9] and to traditional weak PUFs, such as the RO PUF [10], with additional but simple enhancements to expand the challenge-response space from $n$ to $2^{n}$ as required for authentication applications.

The PUF architecture defines a set of functions that convert soft data into bitstrings and keys to be used for encryption and authentication. The authentication protocol that we propose uses soft data and the corresponding Helper Data bitstrings that are produced by the PUF architecture, as input to a correlation technique. We refer to the protocol as Cobra for Correlation-based robust authentication. The results presented in this paper show that by correlating Helper Data bitstrings, a server can correctly and securely authenticate a fielded chip. The significance of this claim is that there is no need to reveal the response bitstrings in the message exchanges between the chip and server and therefore, the traditional approach of applying machine-learning algorithms to the challenge-response-pairs (CRPs) is no longer possible.

In the Cobra protocol, the chip constructs and transmits a Helper Data bitstring to the server. The Helper Data bitstring transmitted is traditionally used by the server to identify weak bits in the response bitstring but otherwise reveals no information about the response bitstring itself [7]. The server constructs a set of Helper Data bitstrings using enrollment data that it stores for all valid chips in a secure database. The chip's Helper Data bitstring is correlated to each of the server generated Helper Data bitstrings by bitwise AND'ing the two bitstrings and then counting the number of ' $1 \mathrm{~s}$ ' in the AND'ed bitstring. (Alternatives to bitwise AND correlation include bitwise XNOR (discussed later) and traditional time and frequency domain digital signal processing forms of correlation.) The server authenticates the chip if exactly one of the Helper Data bitstrings constructed using enrollment data correlates, that is, has a large number of ' 1 's, to the chip's Helper Data bitstring. A similar process is carried out in reverse from server to chip to enable mutual (two-way) authentication but without the exhaustive search component. Therefore, no information regarding the PUF secrets is revealed to the adversary despite the fact that the Helper Data bitstrings are derived from random variations that occur within the PUF's circuit components.

The remainder of this paper is organized as follows. Section 2 provides background on PUF architectures and defines key concepts such as soft data, error avoidance methods, the HELP PUF architecture, Helper Data generation, methods of correlation and an analysis illustrating proof-of-concept. Section 3 describes the Cobra privacy-preserving, mutual authentication protocol. Experimental results are presented in Section 4 on data collected from a set of 500 Xilinx Zynq FPGAs, showing both the effectiveness and the limitations of Cobra on data collected across the range of industrial-level temperature and voltage specifications. A security analysis is presented in Section 5 and conclusions in Section 6. 


\section{Background}

\subsection{PUF Architectures and Soft Data}

A wide range of PUF architectures have been proposed since the initial papers on PUFs were published $[10,11]$. The source of entropy (randomness) for the PUF is chip-to-chip and within-die process variations that occur between and within chips during production. The PUF architecture defines the mechanism that is used to measure small signal variations introduced by process variations effects. In some cases, the measurement process leverages the existing architectural features of the chip, for example, the SRAM PUF measures its entropy source, that is, the state of the individual SRAM cells, by simply applying power to the SRAM array [12]. For most PUFs, however, circuit components need to be added to the chip, for example, the RO PUF requires a set of MUXs and counters to select and measure the frequencies associated with elements in the array of ROs [10]. In another example, the HELP PUF adds a launch-capture clocking mechanism to precisely time the delays of combinational logic paths $[7,8]$.

For PUF architectures that measure and digitize the signal behavior associated with the entropy source, the digitized values provide additional information that can be leveraged in strong challenge-response-pair (CRP) forms of authentication. The digitized values represent the magnitude of the signal behavior, for example, RO frequency or path delay and are often used as input to mathematical processes defined by the PUF architecture. The goal of the mathematical operations is to isolate and amplify the random differences that occur among multiple copies of the individual circuit components. The digitized values are eventually converted to a bit and used in the response of the CRP. We refer to the digitized values as soft data.

Of the PUF architectures that create soft data (the RO and HELP PUFs are just two examples), the conversion to bits can be accomplished in a variety of ways. For example, the RO PUF typically selects a pair of ROs and then computes a difference by subtracting the soft data associated with the two ROs. The sign of the difference can then be used to generate a bit, with, for example, negative differences producing a ' 0 ' and positive differences producing a ' 1 '. The HELP PUF also computes differences among pairings of path delays and uses a modulus operation to assign a ' 0 ' or ' 1 ' to the differences.

\subsection{Error Correction and Avoidance Methods}

Nearly all PUF architecture need to deal with bit-flip errors, which are differences in the response bitstring that occur when the response is regenerated. Bit-flip errors are most probable when the magnitude of the difference between a pair of soft data values is close to zero. In these cases, regeneration of the bitstring, which takes place later in the field and under potentially adverse environmental conditions, can result in bits flipping from ' 0 ' to ' 1 ' or vice versa. Although authentication protocols can be designed to be tolerant to a small number of bit-flip errors, the number of bit-flip errors that can occur is too large in most PUF architectures to guarantee that authentication works correctly when regeneration is carried out in harsh environments.

To deal with this issue, nearly all PUF architectures define an error correction or error avoidance method to improve reliability during regeneration. Error correction is the more popular of the two reliability-enhancing methods. Error correction typically processes the PUF response bits into a final response bitstring using algorithms based on linear block codes [13] or Bose-Chaudhuri-Hochquenghen $(\mathrm{BCH})$ codes [14]. However, nearly all of the error correction schemes ignore the magnitude of the difference in the soft data and use all of the PUF response bits to construct a smaller but reproducible bitstring response, including bits that have a high probability of changing value.

Error avoidance schemes, on the other hand, integrate a thresholding method that skips bits that are deemed unreliable. The reliability of a bit is often directly related to the soft data associated with the bit and in particular, the distance of the soft data value to the bit-flip line. The bit-flip line is defined by the PUF architecture as a soft threshold between ' 0 ' or a ' 1 '. Unlike error correction methods, 
error avoidance methods can only be used with PUF architectures that produce soft data values, for example, the RO [10], metal resistance [15], NVM [16] and HELP $[8,17,18]$ PUFs are some examples. Notable exceptions here are memory-based PUFs, including SRAM, DRAM, FF and latch-based PUFs, which as originally proposed, are not capable to producing soft data.

In typical usage scenarios, an enrollment phase is carried out in which challenges are applied to the PUF in a secure facility and response bitstrings are generated for the first time. In addition to the response bitstring, PUF architectures that use either error correction or error avoidance methods also produce Helper Data. Helper Data is typically maintained in the secure facility and transmitted to the fielded device later during regeneration to enable the PUF to precisely reproduce the response bitstring. The form of the generated Helper Data varies dramatically depending on the error correction or avoidance method employed. A key contribution of the method proposed here relates to Helper Data and in particular to Helper Data that is generated by error avoidance methods. The next two subsections discuss a simple error avoidance scheme used by the HELP PUF as well as features of the generated Helper Data that are leveraged in the proposed Cobra protocol.

\subsection{HELP PUF}

To better exemplify the principles of the proposed technology, we use the HELP PUF architecture and data collected from a set of Zynq FPGAs in the illustrative examples that follow $[17,18]$. HELP uses a launch-capture technique to obtain accurate digital timing values of path delays through a combinational logic block. The combinational logic block for a full adder is shown in Figure 1 but any functional unit can be used (the combinational logic from one column of the Advanced Encryption Standard is used in $[7,8,17,18]$ and for the experimental results presented in Section 4). Logic signal transitions are launched from the Launch FFs shown on the left using $\mathrm{Clk}_{1}$ and captured in the Capture FFs shown on the right using $\mathrm{Clk}_{2}$.
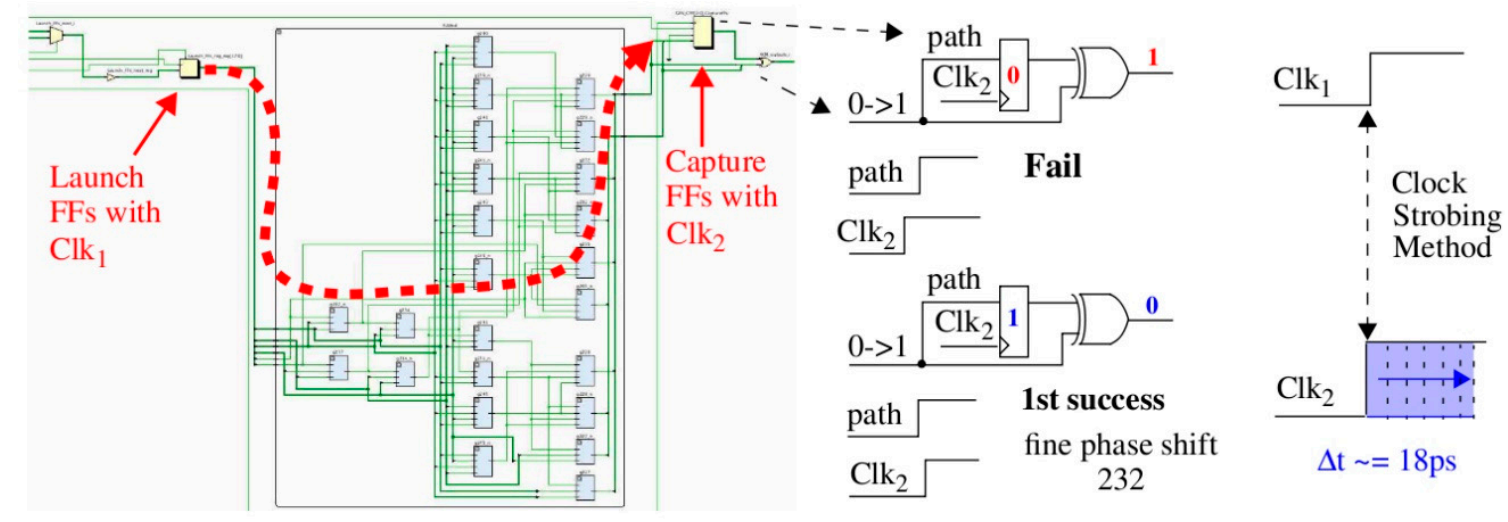

Figure 1. Hardware-embedded delay PUF (HELP) Architecture illustrating Clock Strobing concept used to create path delay (Timing) soft data.

The digital clock manager (DCM) on the FPGA is used to create the clocks, with dynamic fine phase shift enabled for $\mathrm{Clk}_{2}$. Dynamic fine phase shift allows a state machine running in the programmable logic (PL) of the FPGA to increment the phase shift by increments of 18 ps (see right side of Figure 1). A path through the full adder is timed by repeatedly applying a 2-vector sequence to the Launch FFs until the signal propagating along the highlighted path is successfully captured in the Capture FF. A successful capture occurs in this example when the ' 0 ' produced from the first vector $\mathrm{V}_{1}$ of the 2-vector sequence is overwritten by the ' 1 ' produced by $\mathrm{V}_{2}$ (see center portion of Figure 1 ). When this occurs, the current fine phase shift value, which is an integer typically between 100 and 500, is recorded by HELP as the digitized timing value for this path. These timing values represents the soft data associated with HELP. 
During enrollment, a set of timing values, called PUF Numbers or PN, for each chip are stored in the rows of a secure database as shown in Figure 2. This data is consulted by the secure server to authenticate these chips after they are deployed in fielded systems. The storage of soft data on the server, in contrast to response bitstrings, is the first of several significant differences that exist between Cobra and the PUF-based protocols proposed by others [1-6].

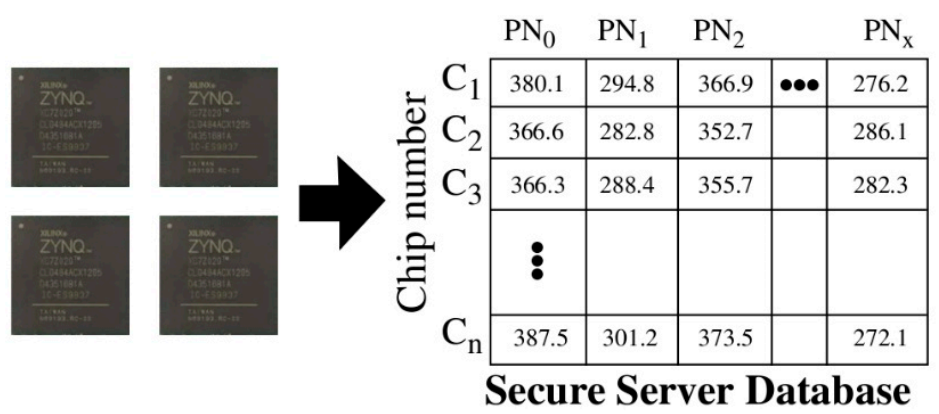

Figure 2. HELP PN database created during enrollment.

As indicated above, the proposed Cobra protocol leverages Helper Data to carry out authentication. The Helper Data is derived from the PNs stored in the secure database on the server and from an instance of HELP that is programmed into the programmable logic of an FPGA, which represents the fielded chip. The entropy that is associated with each chip is captured by the PN stored in the server database. An adversary carrying out machine learning attacks against the protocol would attempt to learn the timing information stored in the database by reverse-engineering the bitstring responses that are exchanged openly over the network. Once learned, the adversary can then impersonate the chip. Therefore, it is vital that the relationship between the PN and the response bitstring be obscured and remain hidden to make this task difficult or impossible for the adversary.

\subsection{Helper Data Generation Using an Error Avoidance Technique}

The illustration presented in Figure 3 shows how bitstrings and Helper Data are generated using an error avoidance scheme called Margining, as a precursor to our discussion on the proposed Helper Data correlation method. The graphs labeled with " $\mathrm{C}_{1}$ " and " $\mathrm{C}_{2}$ " (in large circles) in the center of the figure plot a set of $18 \mathrm{PN}$ (timing values) along the $\mathrm{x}$-axis for two chips $\mathrm{C}_{1}$ (top) and $\mathrm{C}_{2}$ (bottom), with the black curves depicting PN obtained from the secure server database and the red curves depicting PN generated on-the-fly by these chips during regeneration in the field. The environmental conditions for data collected during enrollment and stored in the secure database are specified as $25^{\circ} \mathrm{C}$ under nominal supply voltage conditions $(1.00 \mathrm{~V})$ while to fielded chips are subjected to high temperature $\left(85^{\circ} \mathrm{C}\right)$ and low supply voltage conditions $(-5 \%$ or $0.95 \mathrm{~V})$. The data shown are actual measurements obtained from two Zynq FPGAs used in our experiments.

The HELP PUF converts the PN into a bitstring by applying the following algorithm. First, a pseudo-random number generator selects pairs of $\mathrm{PN}$ and creates differences. A temperature-voltage compensation method called TVComp is then applied to the differences to compensate the measured timing values for changes introduced by environmental conditions (we omit the details of these operations [19] here to focus the discussion on the proposed correlation technique). Finally, a modulus operation is applied to the compensated differences to remove path length bias effects. The modulus operation is defined in the standard way as returning the positive remainder after dividing by the modulus. The value of the modulus is one of several parameters to the HELP algorithm. The graphs shown in Figure 3 use a modulus of 20, which is reflected as the maximum value given on the y-axis.

The TVComp process implemented within HELP is very effective at compensating the chip regenerated PN (red values) but is not ideal. The red data points are vertically offset above and below the black (enrollment) data points because of uncompensated temperature-voltage noise (TVNoise). 
An interesting example labeled 'wrap' is shown for the 2 nd data point in the upper " $\mathrm{C}_{1}$ " graph where TVNoise has caused the point to 'wrap' from the enrollment value near 20 back around to a value near 0 during regeneration. Despite these anomalies, the black and red curves in each graph track very closely, that is, they are correlated. In contrast, the black curves from both graphs (for $C_{1}$ and $C_{2}$ ) are not correlated. This key observation serves as the basis for the innovation proposed within the Cobra protocol.



Environmental conditions: $25^{\circ} \mathrm{C}$ with supply voltage at $1.00 \mathrm{~V}$

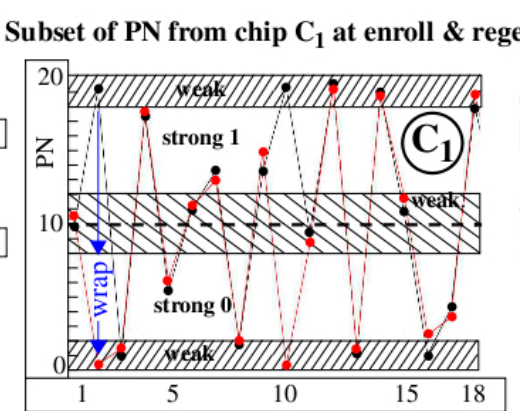

Index of PN for chip $\mathrm{C}_{1}$

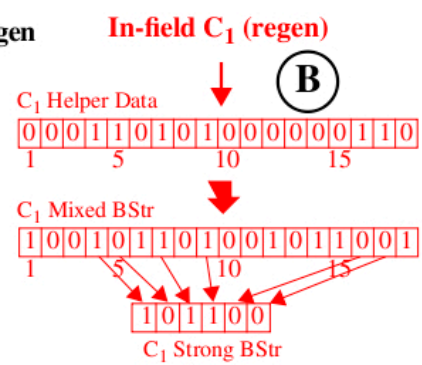

Environmental conditions: $85^{\circ} \mathrm{C}$ with supply voltage at $0.95 \mathrm{~V}$

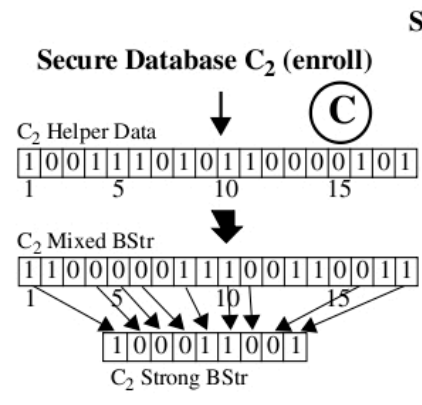

Subset of $P N$ from chip $C_{2}$ at enroll \& regen

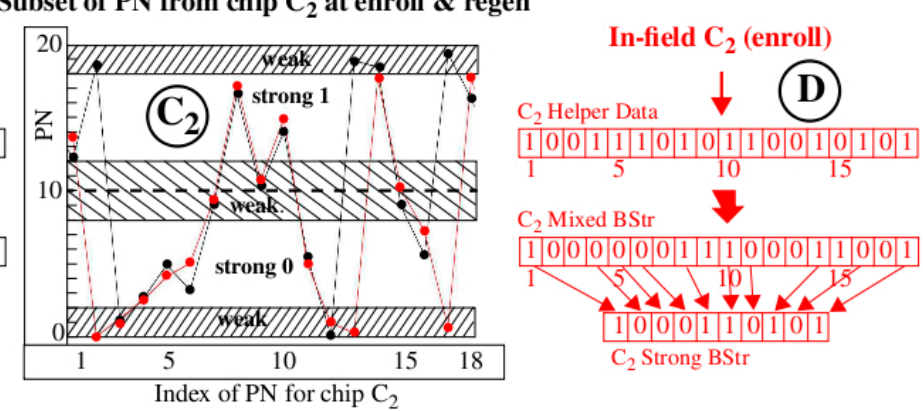

Figure 3. (Center) PN for chips $C_{1}$ and $C_{2}$ under enrollment (black points) and regeneration (red points), conditions, (top left A) Helper Data and response bitstrings for $C_{1}$ under enrollment conditions, (top right B) Helper Data and response bitstrings for $C_{1}$ under regeneration conditions, (bottom left $C$ and right $\mathrm{D}$ ) same for chip $\mathrm{C}_{2}$.

As mentioned earlier, the error avoidance scheme implemented within HELP is called Margining. The Margining scheme skips soft data values (PN in our example) for cases in which the probability of a bit-flip error is large. These highly probable bit-flip regions are labeled "weak" in the center graphs of Figure 3 and are located adjacent to the bit-flip lines at 0,10 and 20. In other words, PN that are within a distance of 2.0 of these bit-flip lines have the highest probability of changing value. For example, the PN labeled "wrap" represents a bit-flip error which is introduced by TVNoise. PN that are located in the "weak" regions are assigned ' 0 ' in the Helper Data bitstring. For example, the " $C_{1}$ Helper Data" bitstrings in the region labeled with the circled " $\mathrm{A}$ " in the figure begins with " 000 ," which reflects that the status of the first $3 \mathrm{PN}$ in the black curve of graph " $\mathrm{C}_{1}$ ". In contrast, the 4 th bit is ' 1 ' because the $\mathrm{PN}$ at position 4 in graph " $\mathrm{C}_{1}$ " falls within the "strong 1 " region.

The " $\mathrm{C}_{1}$ Mixed BStr" in region " $\mathrm{A}$ " of Figure 3 records the bit value associated with each of the $18 \mathrm{PN}$, with $\mathrm{PN}<10.0$ assigned ' 0 ' and those $\geq 10.0$ assigned ' 1 '. This response bitstring corresponds one-to-one to the " $C_{1}$ Helper Data" bitstring and contains both strong and weak bits. The " $C_{1}$ Strong BStr" is constructed from the " $\mathrm{C}_{1}$ Mixed BStr" by selecting only those bits identified as strong in the " $\mathrm{C}_{1}$ Helper Data" bitstring. The region labeled " $\mathrm{B}$ " shows the corresponding bitstrings generated using the red (regeneration) data points from graph " $\mathrm{C}_{1}$ ". The graphs and annotations labeled " $\mathrm{C}_{2}$," " $\mathrm{C}$ " and " $\mathrm{D}$ " are completely analogous to " $\mathrm{C}_{1}$ ", " $\mathrm{A}$ " and " $\mathrm{B}$ " except the $\mathrm{PN}$ and bitstrings are derived from second chip $\mathrm{C}_{2}$. 
The HELP authentication protocol from Reference [7] proposes a DualHelperData scheme in which both the Helper Data and Strong BStr are exchanged between the server modeled on the left side of Figure 3 and the fielded chips modeled on the right side. As discussed earlier, exposing the Strong BStr to the adversary enables model-building attacks where the adversary attempts to reverse engineer the PN stored in the secure database using machine learning (ML) algorithms. Although attempts to model-build HELP have not been successful (see [20]), the exposure of the response bitstrings (Strong BStr) still represents a vulnerability that enables ML attacks. If it becomes possible to construct an ML attack that is able to deduce the relationships among the PN, then the response bitstrings to other challenges can be predicted and the chip impersonated.

\subsection{Proof-of-Concept: Helper Data Correlation}

As a mitigation strategy, we propose an alternative authentication protocol that exchanges only the Helper Data bitstrings. Authentication is carried out in Cobra by correlating the Helper Data bitstrings generated using the enrollment data on the server with the Helper Data bitstring generated on-the-fly by the chip. The simplest correlation strategy is to compute a new bitstring by bitwise AND'ing the Helper Data bitstrings from the server and chip and then counting the number of ' 1 's in the AND'ed version. We refer to the number of ' 1 's as the correlation coefficient (CC) because it reflects the level of similarity that exists (among the ' 1 's) between the two Helper Data bitstrings.

As an example, the left column of Figure 4 shows an authentication attempt by chip $C_{1}$ while the right column shows an attempt by chip $C_{2}$. The top-most red-colored bitstrings in each column are the Helper Data bitstrings transmitted by the chips to the server. For each Helper Data bitstring received during authentication, the server carries out an exhaustive search operation using the PN stored its secure database. It constructs the black-colored Helper Data bitstrings in each column using the technique described in Figure 3 (in fact, the bitstrings shown here are identical to those shown in Figure 3).
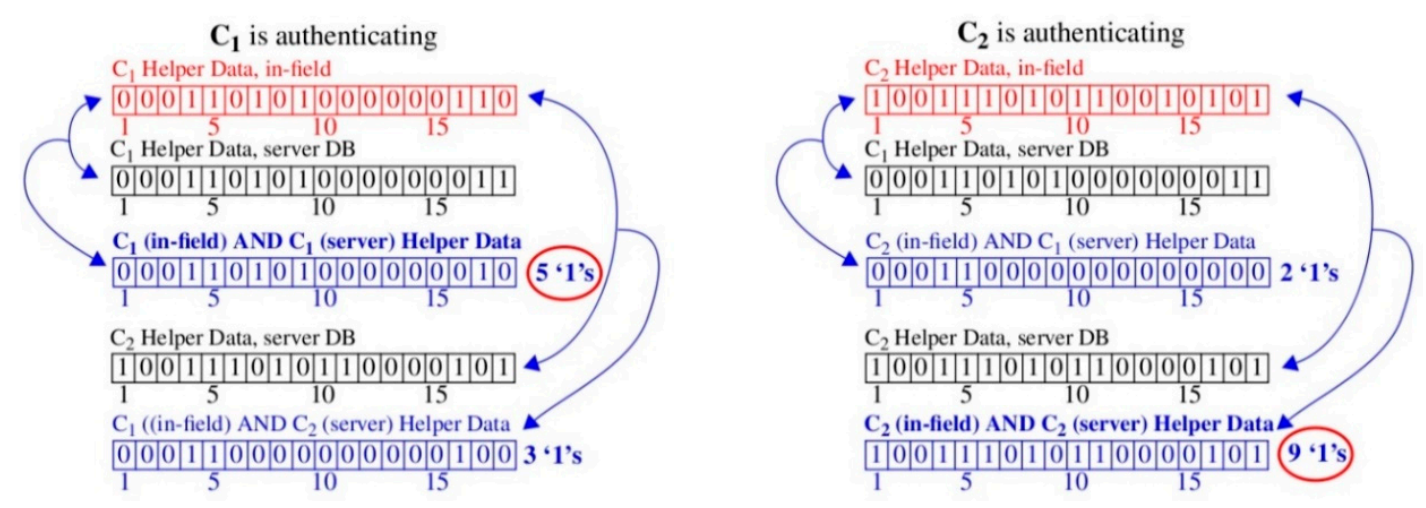

Figure 4. Illustration showing Helper Data bitstring correlation using "AND" operator with $\mathrm{C}_{1}$ authenticating to the server on the left and $C_{2}$ on the right. Helper Data bitstrings are obtained from data in Figure 3.

For each Helper Data bitstring it constructs, the server bitwise AND's it with the received chip's Helper Data bitstring. For example, the AND'ed versions are labeled " $C_{1}$ (in-field) AND $C_{1}$ (server) Helper Data" and " $C_{1}$ (in-field) $A N D C_{2}$ (server) Helper Data" in the left column of Figure 4. As discussed, the bitwise AND operation is a form of correlation that acts to preserve more ' 1 's in cases where the bitstrings are similar. The CCs (number of ' 1 's) are reported to the right of AND'ed Helper Data bitstrings. The results for both authentication attempts show higher correlation for cases where the server-generated Helper Data bitstring is derived using PN collected earlier during enrollment from the same chip. In other words, the authenticating chip is correctly identified to the server using only the information provided by the CC. 
This example uses only a small number of 18 PN from the larger set of 2048 that are produced by each iteration of the HELP algorithm [7]. The results shown in Figure 5 expand the example illustration to the full length bitstrings and to PN collected across 9 TV corners using 500 Xilinx Zynq FPGAs. Each of the 9 curves plots the CCs for the 500 chips along the $x$-axis. The authenticating chip is labeled $\mathrm{C}_{250}$ and is highlighted in the center region of the figure.

The graphic illustration given in Figure 6 shows how the curves in Figure 5 are constructed. Here, the chip's Helper Data bitstring on the left, regenerated under $25^{\circ} \mathrm{C}, 1.00 \mathrm{~V}$, is transmitted to the server on the right. A second authentication request is also shown in red where the chip in this case is regenerating Helper Data with environmental conditions set to $-40{ }^{\circ} \mathrm{C}, 1.05 \mathrm{~V}$. The server carries out an exhaustive search using data stored in the Enroll DB separately for each of these two authentication attempts and computes a set of 500 CCs. The CCs are plotted along the x-axis in Figure 5 as the top-most and bottom-most curves. A similar process is carried out to construct the CCs for the remaining 7 curves in Figure 5 but using PN from the other TV corners.

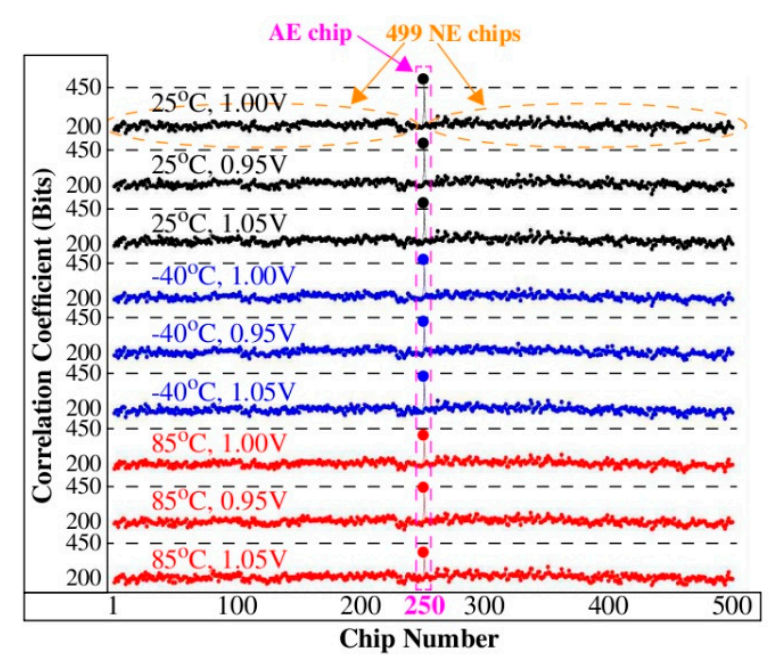

Figure 5. CCs (y-axis) for 500 chips (x-axis) across 9 TV corners obtained by correlating Helper Data bitstrings from the HELP PUF with a Margin of 3 and Modulus of 18. Peak at 250 occurs when Helper Data is derived using PN generated by the chip and matched with PN collected during enrollment for this same chip (called the authentic-enrolled or AE chip). All other CCs are derived by correlating the chip's Helper Data with Helper Data derived using the remaining 499 sets of PN associated with other chips in the Enroll DB (called the non-authentic-enrolled or NE chips).

The CCs in Figure 5 for $\mathrm{C}_{250}$ (referred to as the authentic-enrolled (AE) chip) vary from more than 450 bits in the top curve to approx. 380 bits in the bottom curve. Although the correlation is weakened when the chip is exposed to harsh environmental conditions, it still remains high with respect to the CCs produced by the remaining 499 chips (referred to as non-authentic-enrolled (NE) chips) from the DB. The largest value associated with a NE chip is approx. 260. The large margin between the AE and NE CCs suggests that it should be possible for the server to define a threshold to distinguish successful authentications from unsuccessful authentications with very high probability, for example, any value between 260 and 380 works in this example.

Note that our analysis considers only AE and NE authentication attempts. Two other possibilities include non-authentic-not-enrolled (NN) and non-authentic-counterfeit (NC) authentication attempts. Modeling NN authentication attempts is trivially accomplished by removing their data from the Enroll DB. It follows that attempts to authenticate by chips with no data in the Enroll DB would produce CCs similar to those produced for the 499 NE CCs shown in Figure 5. Modeling NC authentication attempts is difficult without employing some type of machine learning algorithm if in fact an attack model can be devised. We leave this non-trivial task for future work. 


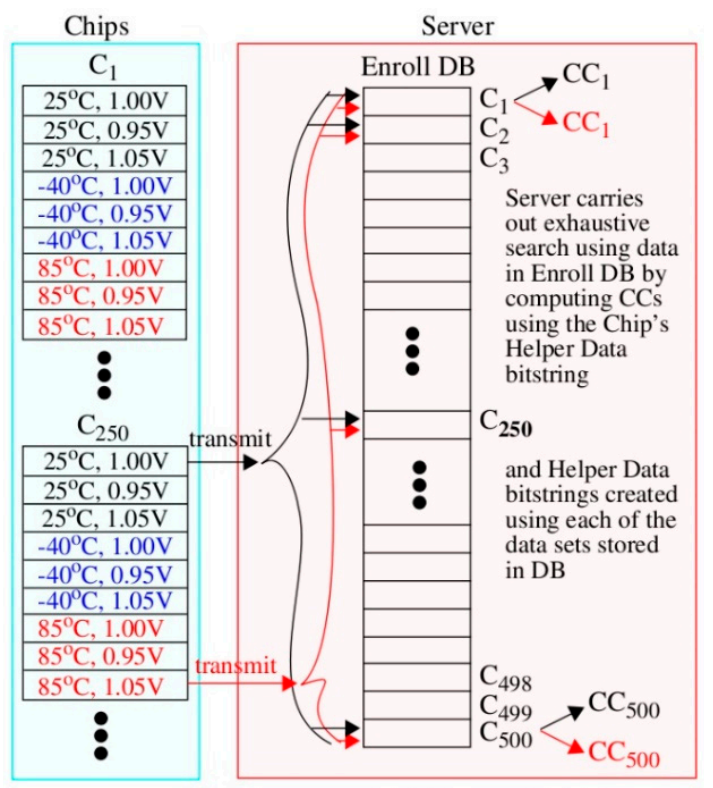

Figure 6. Graphic depicting process used to construct curves shown in Figure 5. Each same-colored set of CCs displayed vertically are concatenated and shown along the x-axis in Figure 5. This process represents exactly what the sever would do to identify and authenticate each Chip's Helper Data bitstring request transmitted to the server. Red-colored CCs correspond to the same $\mathrm{C}_{250}$ authenticating when the environmental conditions are $85^{\circ} \mathrm{C}, 1.05 \mathrm{~V}$ as shown by the bottom-most curve in Figure 5.

We emphasize here that under the AND correlation scheme, it is possible for adversaries to construct Helper Data bitstrings with all ' $1 \mathrm{~s}^{\prime}$ ', which guarantees a large number of matches. However, large CCs would occur for ALL data sets in the secure DB, which, in turn, would be flagged by the server and result in a failed authentication attempt. This is true because the server allows only one CC to be above the threshold in order for an authentication attempt to be classified as successful.

\section{The Cobra Protocol}

In this section, we describe the general structure of the proposed Cobra protocol. A graphical illustration of the Enrollment and Authentication operations, including the message exchanges between the chip and server, are presented in Figure 7 along the top and bottom, respectively. Enrollment is performed in a secure facility using a confidential FPGA programming bitstream that allows access to the PUF's soft data. The server on the right generates challenges $\left\{c_{k}\right\}$ and transmits them to the chip on the left. The chip then applies the challenges to its PUF to generate the set $\left\{\mathrm{PN}_{\mathrm{j}}\right\}$, which is returned to the server. The server generates a chip identifier $I D_{i}$ and stores the soft dataset $\left\{P N_{j}\right\}$ under $I D_{i}$ in its secure database DB.

The first phase of authentication is called Chip Authentication. Here, a fielded chip i requests authentication to the server (note, no chip ID is transmitted to the server in order to preserve privacy). The server generates a nonce $n_{2}$, selects a set of challenges $\left\{c_{m}\right\}$ and transmits them to the chip. The nonce $\mathrm{n}_{2}$ is used to select a Margin parameter and the challenges $\left\{\mathrm{c}_{\mathrm{m}}\right\}$ are a subset of the challenges $\left\{c_{k}\right\}$ used during enrollment. The chip applies the challenges $\left\{c_{m}\right\}$ to its PUF along with a Margin, which is selected using the function ParamSel $\left(\mathrm{n}_{1}\right.$ XOR $\left.\mathrm{n}_{2}\right)$ and generates a Helper Data bitstring $\mathrm{h}^{\prime}$ using the Margining scheme described earlier. Both $\mathrm{h}^{\prime}$ and $\mathrm{n}_{1}$ are transmitted to the server.

The server then carries out a search by processing soft data sets $\left\{\mathrm{PN}_{\mathrm{j}}\right\}_{\mathrm{i}}$ it stores in its database for each chip i. The routine Correlate produces a Helper Data bitstring h internally (not shown) using each of the stored data sets $\left\{\mathrm{PN}_{\mathrm{j}}\right\}_{\mathrm{i}}$ and the same Margin that was used by the chip. The Helper Data bitstring $h^{\prime}$ is then correlated with $h$. Correlation based on a bitwise AND operation was shown in the previous section but other possibilities exist including bitwise XNOR and/or standard waveform 
correlation methods. The output of Correlate is a correlation coefficient $\mathrm{CC}_{\mathrm{i}}$ that is then compared to a threshold t. The authentication is considered successful if exactly one CC is larger than the threshold. The 'exactly one $\mathrm{CC}^{\prime}$ constraint implements a countermeasure against simple adversarial attacks which use Helper Data bitstrings constructed with all 1's. This issue is discussed in detail in Section 5. The threshold is determined in advance using a characterization process in a secure facility. The goal of characterization is to select a threshold that unambiguously distinguishes authentic-enrolled chips (AE) from non-authentic-enrolled (NE), non-authentic-not-enrolled (NN) and non-authentic-counterfeit (NC) chips.

\section{Cobra Protocol}

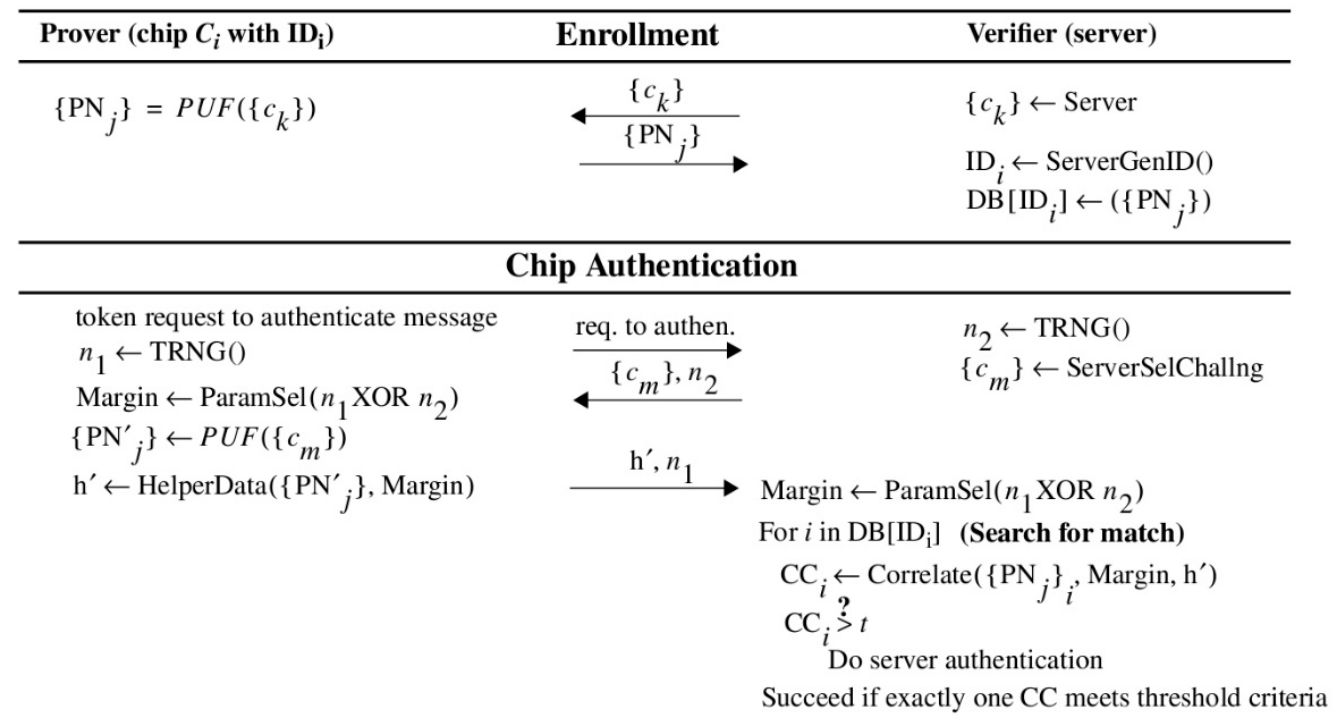

Figure 7. Cobra Protocol: Enrollment (top) and Chip Authentication (bottom) operations and message exchanges of proposed Helper Data bitstring correlation technique for implementing a privacy-preserving, mutual authentication protocol between chip (left) and server (right).

The last phase of the Cobra mutual authentication protocol is for the chip to authenticate the server. This phase is not shown in Figure 7 but is similar except the message exchanges are reversed and the search process is omitted. Moreover, the AND-based correlation scheme used by the server to authenticate the chip cannot be used because the chip does not have access to the secure database. Instead, the chip uses XNOR correlation, which, as we discuss further below, requires matches to both 0 's and 1's in the Helper Data bitstring received from the server and the bitstring produced on-the-fly by the fielded chip.

Server authentication is not performed unless chip authentication succeeds, in which case, the server has identified the chip's soft data set $\left\{\mathrm{PN}_{\mathrm{j}}\right\}_{\mathrm{i}}$. The server uses this $\left\{\mathrm{PN}_{\mathrm{j}}\right\}_{\mathrm{i}}$ to generate another Helper Data bitstring, which is transmitted to the chip. Note that the Helper Data bitstrings generated during server authentication are distinct from those generated during chip authentication because the challenge subset $\left\{c_{m}\right\}$ and nonces $n_{1}$ and $n_{2}$ are selected differently in this phase. Although the nonces select only the Margin parameter in this example, other parameters can be introduced to further expand the CRP space, as described below (and in reference [7]).

\section{Experimental Results}

This section carries out a worst-case analysis using a larger set of the HELP CRP space and discusses the security properties of the Cobra protocol in more detail. Similar to the preliminary analysis presented in Section 2.5, the data analyzed here is collected from a set of 500 chip-instances under enrollment and 9 temperature-voltage (TV) corners. 


\subsection{HELP Challenge Space}

The full CRP space of the HELP algorithm is defined by (1) sets of challenges (2-vector sequences) and corresponding Path-Select-Masks where each challenge set produces 4096 PN and (2) a set of parameters, consisting of two LFSR seeds, two floating point parameters called the reference mean and range and a Modulus and Margin as discussed earlier in Section 2.4. The challenges and Path-Select-Masks create a CRP space with size exponentially related to the size of the functional unit used as the entropy source [21]. The parameters increase the CRP space by approx. $2^{20}$, that is, there are $2^{20}$ 2048-bit bitstrings that can be generated by varying these parameters for each set of challenges and Path-Select-Masks. Only one set of challenges and Path-Select-Masks are used for the analysis carried out in this paper and instead we focus on analyzing Helper Data bitstrings produced by varying only the parameters. Although this represents only a small subset of the entire CRP space, our results show that the Cobra technique works well across a statistically significant sample.

The two LFSR seed parameters allow up to 2048 distinct bitstrings to be generated, each of length 2048 bits. The reference mean and range increase the number of distinct bitstrings by a factor of approx. 128. The analysis performed here analyzes the Helper Data bitstrings generated using 10 distinct LFSR seeds, one combination of the reference mean and range and a set of 11 Moduli and 3 Margins.

\subsection{Illustration of Worst Case Scenario}

The approach taken for the analysis of the worst case is illustrated using Figures 8 and 9. The data presented is derived using only one Modulus and Margin in this section and is expanded to the larger set in Section 4.3. The black curves in Figure 8 plot the CCs computed by the server when each of the $500 \mathrm{AE}$ chips identified along the $\mathrm{x}$-axis authenticates. These curves are constructed as we illustrated for $C_{250}$ in Figure 6 but now include data for all 500 chips and 9 TV corners shown on the left in that figure and for the 10 LFSR seeds. Therefore, there are 90 curves, each with 500 AE CCs. Similarly, the red curves in Figure 8 plot the worst-case (largest) NE CC among the remaining 499 CCs in each authentication attempt while the green curves plot the average NE CC.

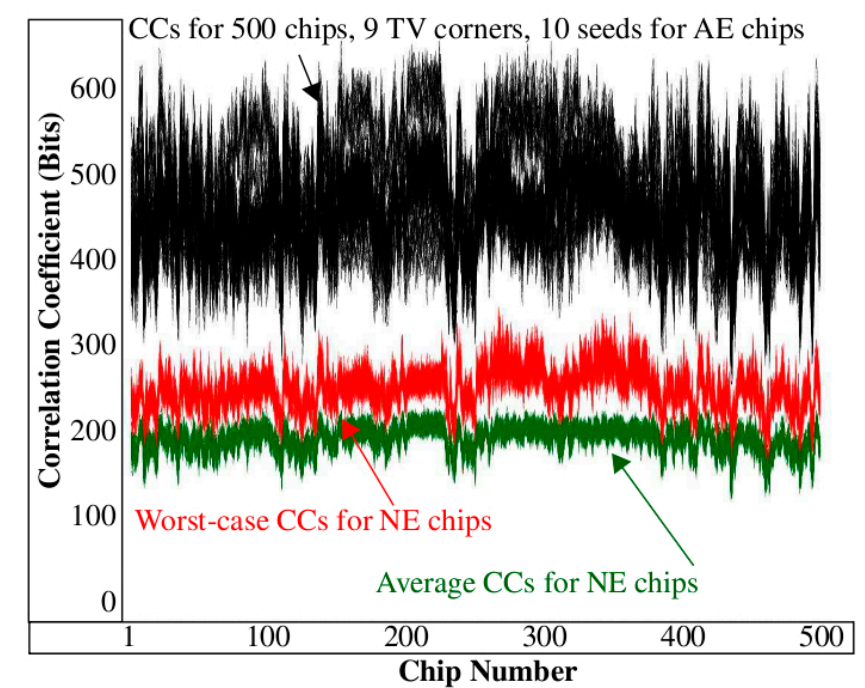

Figure 8. AE chip (black), worst-case NE chip (red), average-case NE chip (green) CCs with a Margin of 3 and Modulus of 18 .

Note that the curves shown in Figure 8 are in a different format than the curves shown in Figure 5. In particular, the CCs in the black curves of Figure 8 correspond to the AE chip only and the red curves plot only one CC (the largest, worst case value) from the 499 NE CCs in each of the curves of Figure 5. Therefore, only two points from each of the curves in Figure 5 are plotted in Figure 8 and appear as column pairs in the black and red curves. The two points in each pair represent the 
worst case (smallest) separation between the AE and NE CCs and visually portray how close a NE chip gets to being falsely authenticated as the AE chip on the server. In summary, the number of black and red CC pairs is given by $500 \times 9$ TV corners $\times 10$ LFSR seeds $=45,000$. Note that each pair corresponds to 500 authentication attempts so in total, there are $45,000 \times 500=22,500,000(22.5 \mathrm{M})$ authentication attempts.

As indicated, the key feature of this graph is the distance (separation) between pairs of points in the black and red curves. This separation is key to the server's ability to distinguish between AE and $\mathrm{NE}, \mathrm{NN}$ and NC chip authentications. Unfortunately, a hard threshold (horizontal line) between the black and red points cannot be drawn without some AE authentications failing (false negatives) and some NE authentications succeeding (false positives).

To deal with this issue, we propose a new metric that computes the percentage change CC, called PCC, as follows. First, a set of CCs are computed using the chip's Helper Data bitstring against each of the server computed Helper Data bitstrings. Then the two largest CCs are identified and plugged in Equation (1) to obtain the PCC value. The server successfully authenticates the chip if the PCC is larger than a hard threshold value and fails otherwise. In cases where the authentication is successful, the soft data in the Enroll DB associated the largest CC, $\mathrm{CC}_{\text {largest}}$, identifies the authenticating chip.

$$
\mathrm{PCC}=\frac{\left(\mathrm{CC}_{\text {largest }}-\mathrm{CC}_{2 \text { nd_largest }}\right)}{\mathrm{CC}_{\text {largest }}}
$$

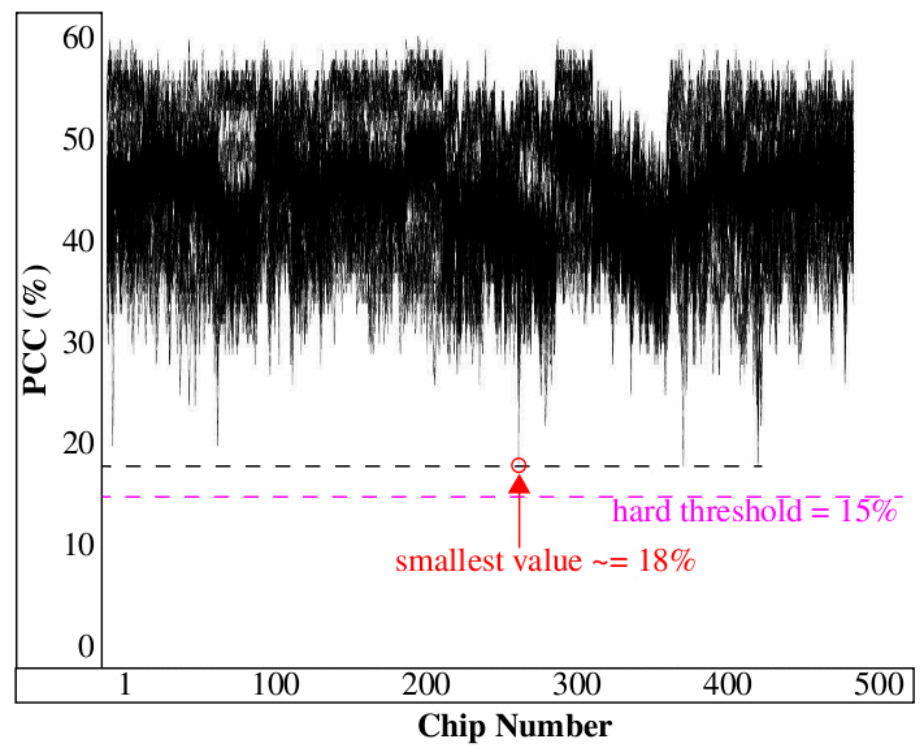

Figure 9. CCs as percentage change using AE chip and worst-case NE chip from Figure 8.

The curves in Figure 9 plot a closely related metric, defined as $\mathrm{PCC}_{\mathrm{AE} \_\mathrm{WC}}$ in Equation (2), using the $\mathrm{CC}$ from Figure 8. Here, $\mathrm{CC}_{\mathrm{AE}}$ is associated with an $\mathrm{AE}$ chip obtained from the black curves in Figure 8 and $\mathrm{CC}_{\text {worst_case_NE }}$ is the other point in the pair obtained from the red curves. Note that in practice, we would not know the authenticate chip and therefore this analysis is somewhat artificial. However, it turns out for every CC pair in Figure $8, C_{\text {largest }}=C_{\mathrm{AE}}$ and $\mathrm{CC}_{2 \text { nd_largest }}=\mathrm{CC}_{\text {worst_case_NE}}$. Therefore, Equations (1) and (2) produce the same results for this set of CCs. In fact, the PCC AE_WC $_{-}$ would be negative if any of the $\mathrm{CC}_{\mathrm{AE}}$ is not the largest $\mathrm{CC}$ among the 500 generated by the server for the authentication attempt. The smallest $\mathrm{PCC}_{\mathrm{AE} \_\mathrm{WC}}$ present is circled in Figure 9 and is approx. $18 \%$. This indicates that all of the $\mathrm{CC}_{\mathrm{AE}}$ are significantly larger than the NE CCs across the $22.5 \mathrm{M}$ authentications. Expressed in terms of bits, the smallest CC from Figure 8 is approx. 300 bits. Therefore, the smallest separation between AE and NE CCs is approx. $300 \times 0.18=54$ bits. A hard threshold can 
now be defined, for example, at $15 \%$ as shown in Figure 9, that enables the server to properly identify and authenticate chips with high probability.

$$
\mathrm{PCC}_{\mathrm{AE} \_\mathrm{WC}}=\frac{\left(\mathrm{CC}_{\mathrm{AE}}-\mathrm{CC}_{\text {Worst_case_NE }}\right)}{\mathrm{CC}_{\mathrm{AE}}}
$$

\subsection{Validation Using the Larger CRP Space}

An analysis reporting PCC $\mathrm{PE}_{\mathrm{AEC}}$ is expanded in this section to a set of 11 Moduli and 3 Margins. The analysis is carried out using bitwise AND correlation as described in the previous sections and is repeated using bitwise XNOR correlation. XNOR correlation further restricts the matching criteria over AND correlation to count matches to both '1's and '0's in the two Helper Data bitstrings. Bitwise XNOR correlation relaxes the criteria used for a successful authentication in the Cobra protocol from 'exactly one' to any CC that exceeds the threshold. This is possible because bitwise XNOR correlation measures the degree of matching between all bits in the Helper Data bitstrings, in contrast to bitwise AND which counts only the number of matching ' 1 's. Interestingly, the two forms of correlation behave differently and are somewhat complementary as we discuss below and further in Section 5.

The PCC $\mathrm{AE}_{-} \mathrm{WC}$ values are shown in Figure 10 for Helper Data bitstrings derived using bitwise AND correlation along the top row and bitwise XNOR correlation along the bottom row. The bar heights represent the worst case $\mathrm{PCC}_{\mathrm{AE}_{-} \mathrm{WC}}$ for a set of Moduli given along the $\mathrm{x}$-axis and Margins given along the $y$-axis. Note that only one $\mathrm{PCC}_{\mathrm{AE} \_W C}$ is reported for each Margin-Moduli combination and in particular, the value that corresponds to the circled point in Figure 9 labeled 'smallest value.' Negative height bars represent that at least one authentication failure has occurred, that is, at least one $\mathrm{CC}_{\mathrm{AE}}$ is not the largest $\mathrm{CC}$ among the $500 \mathrm{CC}$ s computed as we discussed in the previous section.

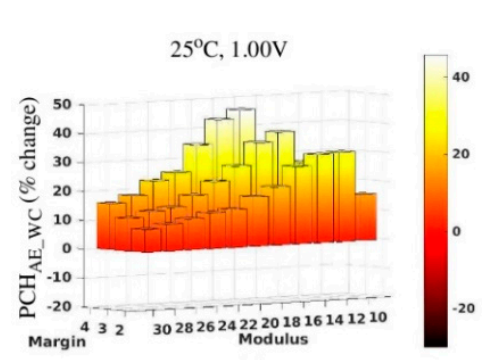

(a)

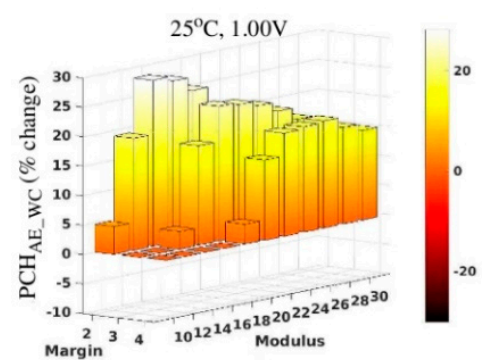

(d)

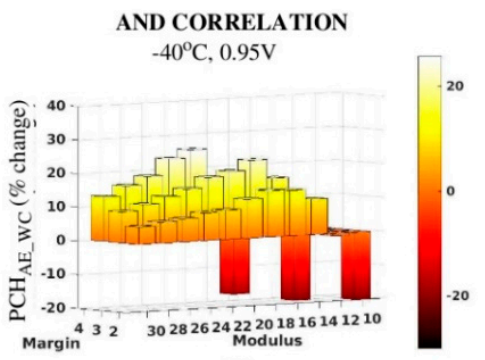

(b)

XNOR CORRELATION $-40^{\circ} \mathrm{C}, 0.95 \mathrm{~V}$

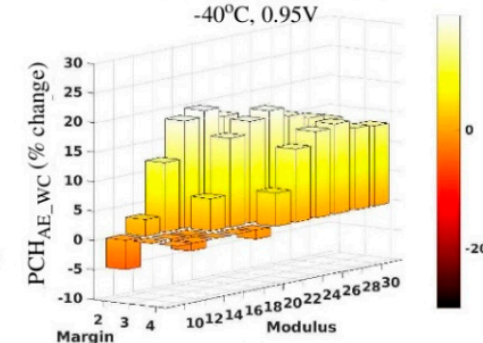

(e)

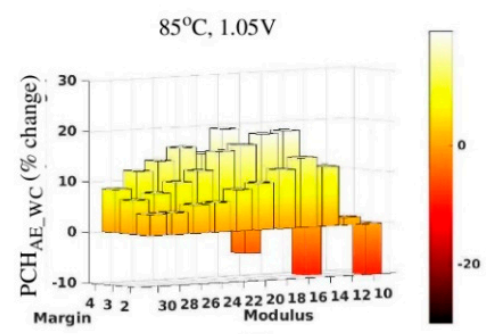

(c)

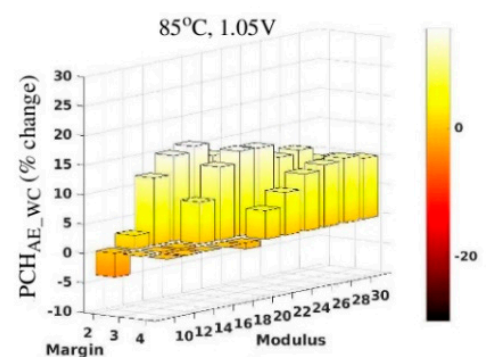

(f)

Figure 10. Worst case Correlation Coefficient (CC) differences between AE and NE chips expressed as percentage change using Equation (2) under nominal environmental conditions, $25^{\circ} \mathrm{C}, 1.00 \mathrm{~V}$ in (a,d) and worst case environmental conditions, $-40{ }^{\circ} \mathrm{C}, 0.95 \mathrm{~V}$ in $(\mathbf{b}, \mathbf{e})$ and $85^{\circ} \mathrm{C}, 1.05 \mathrm{~V}$ in $(\mathbf{c}, \mathbf{f})$ for 22.5 $\mathrm{M}$ authentication attempts for Margins 2 through 4 and Moduli 10 through 30 . Top row gives results using bitwise AND correlation and bottom row gives results using bitwise XNOR correlation. Positive bars indicate server identifies AE chip correctly in all 22.5 M authentications while negative bars indicate at least one false authentication with an NE chip occurred. 
The bar graphs in each of the three columns in Figure 10 gives the results for three TV corners, namely $25{ }^{\circ} \mathrm{C}, 1.00 \mathrm{~V}$ in (a) and (d), $-40{ }^{\circ} \mathrm{C}, 0.95 \mathrm{~V}$ in (b) and (e) and $85^{\circ} \mathrm{C}, 1.05 \mathrm{~V}$ in (c) and (f). The latter two columns represent worst case TV corners, that is, the results for the remaining 6 TV corners (not shown) produce bars that are larger. From these results, it is clear that the Margin-Moduli combinations with negative bar heights cannot be used in the Cobra authentication protocol. However, Moduli between 18 to 22 for Margin 3 and for 22 and 24 for Margin 4 produce bar heights greater than $15 \%$ when using AND correlation. For XNOR correlation, the behavior is reversed regarding the Margin where Margins of 2 and 3 produce better results (note that the bar graph orientation in the top row is rotated $180^{\circ}$ in the bottom row). Here, the PCCs exceed $15 \%$ for Moduli of 16 and 18 for a Margin of 2 and for Moduli 20 and 22 for a Margin of 3.

The bar graphs in the left column for TV corner $25^{\circ} \mathrm{C}, 1.00 \mathrm{~V}$ show the PCCs generated under nominal conditions. Unlike the results for the other TV corners, the bar heights for all Moduli and Margins are positive, indicating that the $\mathrm{CC}_{\mathrm{AE}} \mathrm{S}$ are the largest among the sets of $22.5 \mathrm{M}$ authentications carried out in each analysis.

\section{Security Analysis}

The effectiveness of the proposed Helper Data bitstring correlation method is directly related to two components of the soft data processing algorithm carried out within the PUF architecture. The first is temperature-voltage compensation, referred to as TVComp earlier. Under the HELP algorithm, TVComp scales and shifts the path delays (PN) measured on-the-fly by the chip to make them as similar as possible to the values measured during enrollment under nominal conditions. The proposed correlation techniques depend heavily on the effectiveness of TVComp. For other PUF architectures, for example, the ARB, RO, NVM and metal PUFs, a similar form of TV compensation can be applied as a means of enabling correlation-based authentication as described here for HELP.

A second critically important component of the correlation methods is related to the Margining scheme portrayed within the center graphs of Figure 3. Each of the two response bit regions, with ' 0 ' assigned to the region between 0 and 10 and ' 1 ' assigned to the region between 10 and 20, also contain two strong-weak region boundaries. Bit assignments within the Helper Data bitstrings depend only on these four strong-weak region boundaries and are independent of response bit boundaries at 0 and 10. In other words, a Helper Data bit can be assigned ' 0 ' or ' 1 ' in either of the response bit regions with equal probability. The symmetrical placement of the strong-weak region boundaries within each response bit region eliminates leakage in the Helper Data bitstrings and is the basis for the claimed improvements to model-building resistance of the correlation methods.

As mentioned earlier, when the AND correlation method is used by Cobra to generate PCCs, an authentication is deemed successful only in cases where exactly one server-computed PCC is above the threshold. The requirement of 'exactly one PCC' represents a countermeasure to adversarial attacks in which Helper Data bitstrings are artificially constructed with all ' 1 's or a large fraction of ' 1 's. The server is effectively screening for an outlier, that is, one PCC that is significantly larger than all the others it computes during the exhaustive search process. A successful impersonation attack then requires the adversary to construct a Helper Data bitstring that is consistent with a matching server-generated version at all bit positions, that is, both the ' 0 's and ' 1 's must be correlated. Otherwise authentication fails because more than PCC is above the threshold.

From the results shown in Figure 10a, the AND correlation method performs best, that is, produces the largest PCCs, when the fraction of ' 1 's in the Helper Data bitstrings is small. The fraction of ' 1 's (and ' 0 's) is determined by the ratio of the Margin and Modulus. The Margining scheme requires the Modulus $\geq 4 \times$ Margin +2 . As an example, when the Margin is 4 , the Modulus must be at least 18. Assuming the PN are evenly distributed across the range defined by the Modulus (which is not valid for individual PN but is valid across a large collection of PN), the fraction of '1's is approximately equal to the sum of the two strong bit regions divided by the Modulus. For AND correlation, the best results are obtained when the strong response bit regions are size 1 or 2 . In particular, the largest 
$\mathrm{CC}_{\mathrm{AE}}$ is nearly $45 \%$ larger than $\mathrm{CC}_{\text {worst_case_NE }}$ in Figure 10 a for a Margin of 4 and a Modulus of 18 . The fraction of ' 1 's in this case is $2 / 18 \cong 11 \%$.

The strong-weak bit regions act as selection functions for the correlation methods. The AND correlation method is a one-sided selection function because only the 'strong bit' side of the boundary impacts the PCC. From the results and correlation performs best when the selection regions are asymmetrically skewed toward narrow strong bit regions.

In contrast, both sides of the strong-weak boundaries affect the XNOR PCC. This fundamental difference in the two correlation functions is reflected in the PCCs computed using different Margins. For AND correlation in Figure 10a, the largest PCCs occur using a Margin of 4 for the majority (not all) of the Margin-Moduli combinations, while best case for the XNOR correlation occurs for a Margin of 2 . The bar graphs in the second row are rotated $180^{\circ}$ to more clearly illustrate this characteristic. For example, the best results for XNOR occur for a Margin of 2 and for Moduli of 14 and 16. For these Margin-Moduli combinations, the size of weak and strong bit regions are nearly equal (they are equal for a Modulus of 16). Therefore, the two-sided XNOR selection function appears to work better for Margin-Moduli combinations that divide the entire region more evenly into weak and strong regions. Given the distinctive behavior of the AND and XNOR correlation functions, the Cobra protocol can in fact leverage both PCCs as a means of reducing the probability of false negative and false positive authentication decisions.

The overall decrease in the PCC magnitudes under adverse environmental conditions (Figure 10b,c,e,f) is caused by TVNoise. From the results, it is clear that the level of sensitivity of the PCCs to TVNoise is higher for smaller Margins and Moduli.

\subsection{Overhead of the Cobra Protocol}

The largest component of the overhead associated with the Cobra protocol is related to the PUF architecture. In Reference [18], Table 2 gives the resource requirements for the HELP algorithm and original HELP protocol as 2350 LUTs and 1454 FFs with an additional 706 and 3494 LUTs needed to implement the functional unit (entropy source) using an instance of AES SBOX and AES sbox_mixcol, resp. An instance of sbox_mixcol is used to generate the data presented in this paper. It consists of 4 AES SBOXs and 132-bit copy of AES mixed column implemented in a hazard-free combinational logic style. Cobra eliminates the need to generate the response bitstring and therefore, is slightly smaller by approx. 100 LUTs. For example, the size of the implementation used for experiments in this paper is 5750 LUTs and $1454 \mathrm{FFs}$, excluding the LUTs and FFs used by the Xilinx IP. Total size with Xilinx IP is 6770 LUTs and 3271 FFs, plus 1 MMCM and 125 -bit multiplier.

\subsection{Analysis of Cobra's Challenge-Response Space}

As outlined earlier in Section 4.1, the full CRP space of HELP is defined by two components; (1) the values associated with a set of six parameters including 2 11-bit LFSR seeds, two floating point parameters called the reference mean and range and a Modulus and Margin and (2) the challenges and Path-Select-Masks. The challenges and Path-Select-Masks are used to select two sets of 2048 PN from the larger set $\left\{\mathrm{PN}_{\mathrm{j}}\right\}$ stored in the secure database (see Figure 7). The number of distinct 2048-bit Helper Data bitstrings that can be generated by varying the parameters is given by the product: $2048 \times 128 \times$ $2 \times 2=2^{20}$ or 1 million. HELP first creates 2048 PN differences (PND) by subtracting unique pairs of elements from the two sets of 2048 PNs. The factor 2048 in the above product represents the number of ways unique sets of 2048 PND can be created using the LFSR seeds. HELP applies a procedure called TVComp that shifts and scales the PND using two floating point parameters called the reference mean and range. The factor 128 in the product represents a conservative estimate on the number of ways the PND can be modified to produce unique response and Helper Data bitstrings by varying these parameters. Finally, the factors of 2 represent a conservative estimate on the number of Margins and Moduli that can be applied, as we discussed in Section 4.2. Note that $2^{20}$ represents the number of 
unique Helper Data bitstrings that can be produced using the 4096 PN selected by one set of challenges and Path-Select-Masks.

The challenges and Path-Select-Masks represent the larger component of the CRP space. In [21], we used two sets of $7500 \mathrm{PN}$ as the enrollment data (15,000 PN), where each PN can be stored as a 16-bit fixed-point value, resulting in less than $32 \mathrm{~KB}$ of storage in the secure database per fielded device. Although the number of distinct PND that can be created from the two sets of $7500 \mathrm{PN}$ is $7500^{2} \cong 56$ million, the number of distinct response and Helper Data bitstrings that can be produced is much larger because of the Distribution Effect (which is the main topic of [21]). The challenges and Path-Select-Masks allow any two subsets of 2048 PN from the 7500 sets to be selected. The Distribution Effect is an artifact of the TVComp process, which transforms any given PND into one of approximately 100 different compensated PND $\left(\mathrm{PND}_{\mathrm{c}}\right)$. More importantly, it is not possible to predict the value of a $\mathrm{PND}_{\mathrm{c}}$ unless the entire set of $2048 \mathrm{PND}$ are known. Although the Distribution Effect only increases the number of distinct $\mathrm{PND}_{\mathrm{c}}$ to approx. 5.6 billion, the number of different distributions of 2048 PND is characterized as (7500 select 2048) which is a very large exponential.

The entire CRP space with 15,000 PN per fielded device would then be lower bounded by the product of $2^{20} \times 2^{32}=2^{52}$, which accounts for both the parameters and challenge components. The large diversity of HELP's CRP space prevents replay attacks and adds significantly to the difficulty of model-building attacks if in fact such attacks are possible using only Helper Data bitstrings.

\section{Conclusions}

A privacy-preserving, mutual PUF-based authentication protocol called Cobra is described in this paper. Cobra exchanges and correlates Helper Data bitstrings instead of PUF response bitstrings as a means of authenticating the chip and server. Helper Data is derived from the PUF response bitstrings and therefore the Helper Data bitstrings inherit the randomness and uniqueness characteristics associated with the PUF's source of entropy. By eliminating PUF response bitstrings in the message exchanges between the chip and server, attacks such as model-building are much more difficult to carry out. Cobra is demonstrated on a statistically significant set of FPGAs using the HELP algorithm and a simple thresholding method is proposed that the server and chip can use for authentication. Although the HELP algorithm is used in this paper, the method is applicable to any PUF that produces soft data, that is, digitized values that capture the magnitude of signal behavior such as delay or metal resistance. Future work will investigate the application of the Helper Data correlation method to other PUF architectures and will evaluate more traditional forms of correlation that are used in digital signal processing applications.

Author Contributions: Conceptualization, J.P.; Methodology, J.P.; Validation, M.A.; Writing-Original Draft Preparation, J.P. and M.A.

Funding: This research received no external funding.

Conflicts of Interest: The authors declare no conflict of interest.

\section{References}

1. Bolotnyy, L.; Robins, G. Physically Unclonable Function-based Security and Privacy in RFID Systems. In Proceedings of the Fifth Annual IEEE International Conference on Pervasive Computing and Communications (PerCom'07), White Plains, NY, USA, 19-23 March 2007; pp. 211-220.

2. Hammouri, G.; Ozturk, E.; Sunar, B. A Tamper-Proof and Lightweight Authentication Scheme. Pervasive Mob. Comput. 2008, 4, 807-818. [CrossRef]

3. Sadeghi, A.-R.; Visconti, I.; Wachsmann, C. Enhancing RFID Security and Privacy by Physically Unclonable Functions. In Towards Hardware-Intrinsic Security; Information Security and Cryptography; Springer: Berlin/Heidelberg, Germany, 2010; pp. 281-305.

4. Kocabas, U.; Peter, A.; Katzenbeisser, S.; Sadeghi, A. Converse PUF-Based Authentication. In Trust and Trustworthy Computing; Springer: Berlin/Heidelberg, Germany, 2012; pp. 142-158. 
5. Majzoobi, M.; Rostami, M.; Koushanfar, F.; Wallach, D.S.; Devadas, S. Slender PUF Protocol: A Lightweight, Robust, and Secure Authentication by Substring Matching. In Proceedings of the 2012 IEEE Symposium on Security and Privacy Workshop, San Francisco, CA, USA, 24-25 May 2012; pp. 33-44.

6. Aysu, A.; Gulcan, E.; Moryama, D.; Schaumont, P. Compact and Low-power ASIP Design for Lightweight PUF-based Authentication Protocols. IET Inf. Secur. 2016, 10, 232-241. [CrossRef]

7. Che, W.; Martin, M.; Pocklassery, G.; Kajuluri, V.K.; Saqib, F.; Plusquellic, J. A Privacy-Preserving, Mutual PUF-Based Authentication Protocol. Cryptography 2017, 1, 3. [CrossRef]

8. Aarestad, J.; Ortiz, P.; Acharyya, D.; Plusquellic, J.F. HELP: A Hardware-Embedded Delay-Based PUF. Des. Test Comput. 2013, 17-25. [CrossRef]

9. UNM Publication. Available online: http://ece-research.unm.edu/jimp/pubs/ARB_PUF.pdf (accessed on 30 March 2013).

10. Gassend, B.; Clarke, D.E.; van Dijk, M.; Devadas, S. Silicon Physical Unknown Functions. In Proceedings of the ACM Conference on Computer and Communications Security, Washington, DC, USA, 18-22 November 2012; pp. 148-160.

11. Lofstrom, K.; Daasch, W.R.; Taylor, D. IC Identification Circuits using Device Mismatch. In Proceedings of the International Solid State Circuits Conference, San Francisco, CA, USA, 9 February 2000; pp. 372-373.

12. Guajardo, J.; Kumar, S.S.; Schrijen, G.-J.; Tuyls, P. FPGA Intrinsic PUFs and their use for IP Protection. In Proceedings of the Cryptographic Hardware and Embedded Systems (CHES), Vienna, Austria, 10-13 September 2007; pp. 63-80.

13. Dodis, Y.; Reyzin, L.; Smith, A. Fuzzy Extractors: How to Generate Strong Keys from Biometrics and Other Noisy Data. In Proceedings of the Advances in Cryptology (EUROCRYPT), Interlaken, Switzerland, 2-6 May 2004; pp. 523-540.

14. Bosch, C.; Guajardo, J.; Sadeghi, A.-R.; Shokrollahi, J.; Tuyles, P. Efficient Helper Data Key Extractor on FPGAs. In Proceedings of the Workshop Cryptographic Hardware and Embedded Systems (CHES), Washington, DC, USA, 10-13 August 2008; pp. 181-197.

15. Helinski, R.; Acharyya, D.; Plusquellic, J. A Physical Unclonable Function Defined Using Power Distribution System Equivalent Resistance Variations. In Proceedings of the Design Automation Conference, San Francisco, CA, USA, 26-31 July 2009; pp. 676-681.

16. Che, W.; Bhunia, S.; Plusquellic, J. A Non-Volatile Memory based Physically Unclonable Function without Helper Data. In Proceedings of the International Conference on Computer-Aided Design, San Jose, CA, USA, 3-6 November 2014.

17. Che, W.; Kajuluri, V.K.; Martin, M.; Saqib, F.; Plusquellic, J.F. Analysis of Entropy in a Hardware-Embedded Delay PUF. Cryptography 2017, 1, 8. [CrossRef]

18. Che, W.; Saqib, F.; Plusquellic, J. Novel Offset Techniques for Improving Bitstring Quality of a Hardware-Embedded Delay PUF. IEEE Trans. Very Large Scale Integr. (VLSI) Syst. 2018, 26, 733-743. [CrossRef]

19. See Video Tutorials Labeled 'HELP' and 'HELP Protocol'. Available online: http:/ / ece-research.unm.edu/ jimp/HOST/index.html (accessed on 1 April 2018).

20. Che, W.; Martinez-Ramon, M.; Saqib, F.; Plusquellic, J. Delay Model and Machine Learning Exploration of a Hardware-Embedded Delay PUF. In Proceedings of the Symposium on Hardware-Oriented Security and Trust (HOST), Washington, DC, USA, 1-7 May 2018.

21. Che, W.; Kajuluri, V.K.; Saqib, F.; Plusquellic, J. Leveraging Distributions in Physical Unclonable Functions. Cryptography 2017, 1, 17. [CrossRef]

(C) 2018 by the authors. Licensee MDPI, Basel, Switzerland. This article is an open access article distributed under the terms and conditions of the Creative Commons Attribution (CC BY) license (http:// creativecommons.org/licenses/by/4.0/). 